\title{
Morphological and Filtration Performance Studies of Alumina Disc Membranes
}

\author{
'Azzah Nazihah Che Abdul Rahim , 'Ainun Sailah Sihar', Munawar Zaman Shahruddin', Nur Hashimah Alias ', \\ Siti Nurliyana Che Mohamed Hussein ${ }^{1}$, Mohd Fazril $\operatorname{Irfan}^{1}$,Nur Hidayati Othman ${ }^{1 *}$ \\ ${ }^{I}$ Department of Oil and Gas Engineering, Faculty of Chemical Engineering, UiTM, 40450, Shah Alam, Selangor, Malaysia \\ *Corresponding author E-mail: nurhidayati0955@salam.uitm.edu.my
}

\begin{abstract}
Ceramic membranes can be used for water separation and gas filtration owing to its chemical inertness, good mechanical and porous properties. In this work, a total of 18 ceramic membranes were fabricated by varying the parameters such as ceramic powder composition (Al2O3), solvent (DMSO) concentration in external coagulation bath, and sintering time. The morphologies of the prepared alumina disc membranes prepared were studied using digital microscope. In order to understand the effects of morphologies toward the perme ation rate of the ceramic membranes-dead end water permeation tests and salt rejection tests were carried out to study the performance of the alumina disc membranes. Membrane 1 which was fabricated with $50 \%$ alumina loading, $0 \%$ DMSO in coagulant and sintering time of $8 \mathrm{~h}$ was used as reference membrane. It was found that by increasing the alumina up to $65 \%$, the structure become denser and water flux decreased up to $26 \%$, while the salt rejected increased by $55 \%$. Effect of increasing the solvent concentration in external coagulation bath was obvious in finger length which becomes shorter and performed $61 \%$ but fluctuates in salt rejection. Sintering time effect was not significant in salt rejection studies as it gives only small difference but increase in water flux because the longer sintering time caused void to the membrane. Different results were shown and proven with varied parameters such as alumina loading, DMSO concentration and sintering time.
\end{abstract}

Keywords: Ceramic membranes; alumina discs; morphology; filtration; water treatment.

\section{Introduction}

Ceramic membranes are considered as a viable alternative to polymeric membranes in wastewater treatment processes owing to their high chemical, thermal, and mechanical resistance. They are generally more resistant to harsh operating conditions, thus, minimizing the operating cost. Isostatic pressing is commonly used to manufacture ceramic membranes [1]. However, it is quite difficult to tailor the structure and morphology of the fabricated membranes.

Phase inversion is a controlled process of polymer transformation from a liquid phase to solid phase and has been widely exploited in the fabrication of polymeric membranes [2]. The formation of various membrane structures/morphology, especially the formation of "skin layer" is as a result of thermodynamic and kinetic aspects of phase separation processes. The use of phase inversion and sintering technique for the fabrication of ceramic membranes has recently emerged due to their relatively simple laboratoryscale fabrication process. The fabrication method of using single step of inducing phase inversion and sintering technique have greatly reduced the energy and time consumed. A reduction in the number of steps was observed as compared to traditional pressing technique by eliminating the needs of additional deposit layer on the substrate to produce microfiltration or ultrafiltration membranes [3]. This makes the fabrication of ceramic membrane to be more effective and have attracted researchers to produce ceramic membranes for wide range of applications.
Fabrication via this method promises asymmetric structure, which consists of finger-like and sponge like region due to the phenomenon which occurred at the interface between two different viscosity fluids when it first met. Ceramic suspension from the mixing of ceramic/polymer/solvent once immersed in a non-solvent (coagulant) will cause a steep concentration gradient that led to the exchange of solvent and non-solvent. This will result in the precipitation of polymer phase while the ceramic particle is immobilized. The instabilities at the interface between the alumina suspension and coagulant is the initiating factor for viscous finger-like to form. The thickness and length of the finger-like and sponge-like region has great impact on the properties of the membrane and the effort to understand the mechanisms are crucial to produce membrane with desired application. The present work is therefore aimed at examining how the parameters of choice affects the formation and the morphology of membranes.

In this study, the effects of alumina ceramic composition and coagulation bath compositions towards the membrane morphology and filtration performances were investigated. The fabricated alumina disc membranes were characterized by digital microscope and the filtration performance was investigated using sodium chloride solution. 


\section{Experimental}

\subsection{Chemicals and Materials}

Alumina oxide (Al2O3) with an average size of 1.0 micron surface area $5.6 \mathrm{~m}^{2} / \mathrm{g}$ were obtained from Alfa Aeser and used as a ceramic powder for the dope preparation. Polyethersulfone (PESf) was obtained from (Solavay Advanced Polymer, Belgium), Arlacel P135 (Polyetyleneglycol-30-dipolyhydroxystearate (Uniqema, USA) and Dimethyl Sulfoxide (DMSO) (Merck, USA) were used as polymer binder, solvent and additive, respectively. Tap water was used as coagulants and sodium chloride $(\mathrm{NaCl})$ obtained from J. T. Baker was used during the filtration performance study.

\subsection{Fabrication of Alumina Disc Membrane}

A series of dope suspension were prepared according to formulation in Table 1. First, 0.38 wt $\%$ of Arlacel P135 was dissolved in a pre-determined amount of DMSO. Then, $\mathrm{Al}_{2} \mathrm{O}_{3}$ powder was added to the homogenous suspension before roll-milled for $48 \mathrm{~h}$ with 20 $\mathrm{mm}$ agate milling ceramic balls. Milling was continued for another $48 \mathrm{~h}$ after the addition of PESf to obtain homogenous dope suspension.

Table 1: Dope suspensions formulation

\begin{tabular}{|l|c|c|c|}
\hline \multirow{2}{*}{ Materials } & \multicolumn{3}{|c|}{ Abbreviation/ Composition (wt \%) } \\
\cline { 2 - 4 } & $50 \mathrm{ADM}$ & $57.5 \mathrm{ADM}$ & $65 \mathrm{ADM}$ \\
\hline Alumina (Ceramic powder) & 50.00 & 57.50 & 65.00 \\
\hline Arlacel (Dispersion) & 0.38 & 0.38 & 0.38 \\
\hline DMSO (Solvent) & 44.62 & 36.37 & 28.12 \\
\hline PESfv(Polymer) & 5.00 & 5.75 & 6.50 \\
\hline
\end{tabular}

The disc membranes were fabricated by pouring a pre-degassed dope suspension into $1 \mathrm{~mm}$ thickness and $30 \mathrm{~mm}$ diameter stainless steel mould. The mould was then horizontally placed in a coagulation bath of $0 \mathrm{wt} \%$ solvent (pure tap water). The mould was left in the coagulation bath for 1 hour and after the pre-cursor alumina disc was formed, the mould was removed. Similar step was repeated by using different solvent composition in coagulation bath which were $60 \%$ and $80 \%$ leading to 5 types of membranes.

The alumina disc precursor was them calcined and sintered (MXI Hipan) in order to form final alumina disc membranes (ADMS). 2 steps sintering profile was used. First, the temperature of furnace was increased from room temperature to $600{ }^{\circ} \mathrm{C}$ at a rate of $2{ }^{\circ} \mathrm{C} / \mathrm{min}$ and held for 2 hours and further increased to the target temperature of $1450^{\circ} \mathrm{C}$ at a rate of $5^{\circ} \mathrm{C} / \mathrm{min}$ and held for 8 hours

\subsection{Morphology Observation and Performance Study}

The cross-section of disc images were collected using digital microscope. A green dye was used to clearly observe the morphology as the disc membranes are white in color.

The filtration system for salt rejection were set up as our previous work [4]. The membranes were sealed to the end of the tube using epoxy resin. For salt rejection study, sodium chloride $(\mathrm{NaCl})$ was used as solute. The feed tank was filled up with $7000 \mathrm{~mL}$ of $1000 \mathrm{ppm}$ saltwater. The feed was continuously supplied to disc modules for 15 min to stabilize the pressure at 2 bar. The time taken for the collected water to reach $10 \mathrm{ml}$ were recorded and the conductivity value was recorded using conductivity meter. The performances of ADMs were evaluated based on permeate flux and salt rejection rate. The permeate flux $(J)$ is calculated by using the following equation:

$J=\frac{W}{A t}$ where $W$ is the permeate volume $(L), A$ is the effective membrane area $\left(\mathrm{m}^{2}\right)$ and $t$ is the interval time $(h)$. The salt rejection efficiency is calculated by using the following equation:

$R=\left[1-\frac{C p}{C f}\right] \times 100 \%$

where $C_{p}$ and $C_{f}$ are concentration of sodium chloride in permeate and feed solution (ppm), respectively. The oil concentrations in the permeate and in the feed were determined by conductivity meter.

\section{Results and Discussion}

\subsection{Morphological Studies}

Figure 1 shows the digital microscope images of alumina disc membranes prepared in this work. 50ADM0 was used as reference membrane and sintered for 8 hours at $1450{ }^{\circ} \mathrm{C}$. It can be seen that as the alumina loading in the dope suspension increased, the ADM structure becomes denser. For 50ADM0, a typical asymmetric structure that consists of finger-like region at the bottom of the alumina disc and sponge like region at the top of it is observed. Almost $80 \%$ of the alumina disc region is covered by finger-like macro void. Similar structure was observed when DMSO was used as the solvent (Lee at al., 2016, Kinsgbury). This might be due to phase inversion between the solvent in the dope suspension and non-solvent (water) in the coagulation bath. Interestingly, when the alumina loading was increased to $57.5 \%$ and then to $65 \%$, the finger-like region immediately disappeared forming a symmetric sponge-like / dense structure. The alumina disc formed a two-thin sponge-like layer sandwiching when using $57.5 \%$ alumina composition (57.5ADM0) and 100\% dense with 65\% alumina loading (60ADM0). This indicates that the loading of alumina plays a significant role in determining the final structure of the fabricated membrane.

Altering the formulation of suspension to $65 \%$ caused the increased of alumina/PESf ratio, thus the dope suspension viscosity. This directly affects the formation of finger-like structure. Less viscous dope led to formation of finger-like structure due to the effects of hydrodynamically unstable viscous fingering phenomenon that occurred simultaneously when two fluid that have different viscosity are in contact. Moreover, the polymer that is present in the alumina suspension are miscible with water, hence the exchange of solvent and non-solvent occurred rapidly. The increment of viscosity in this study was proven to cause the membrane to inhibit finger-like formation and instead forming a dense membrane. 


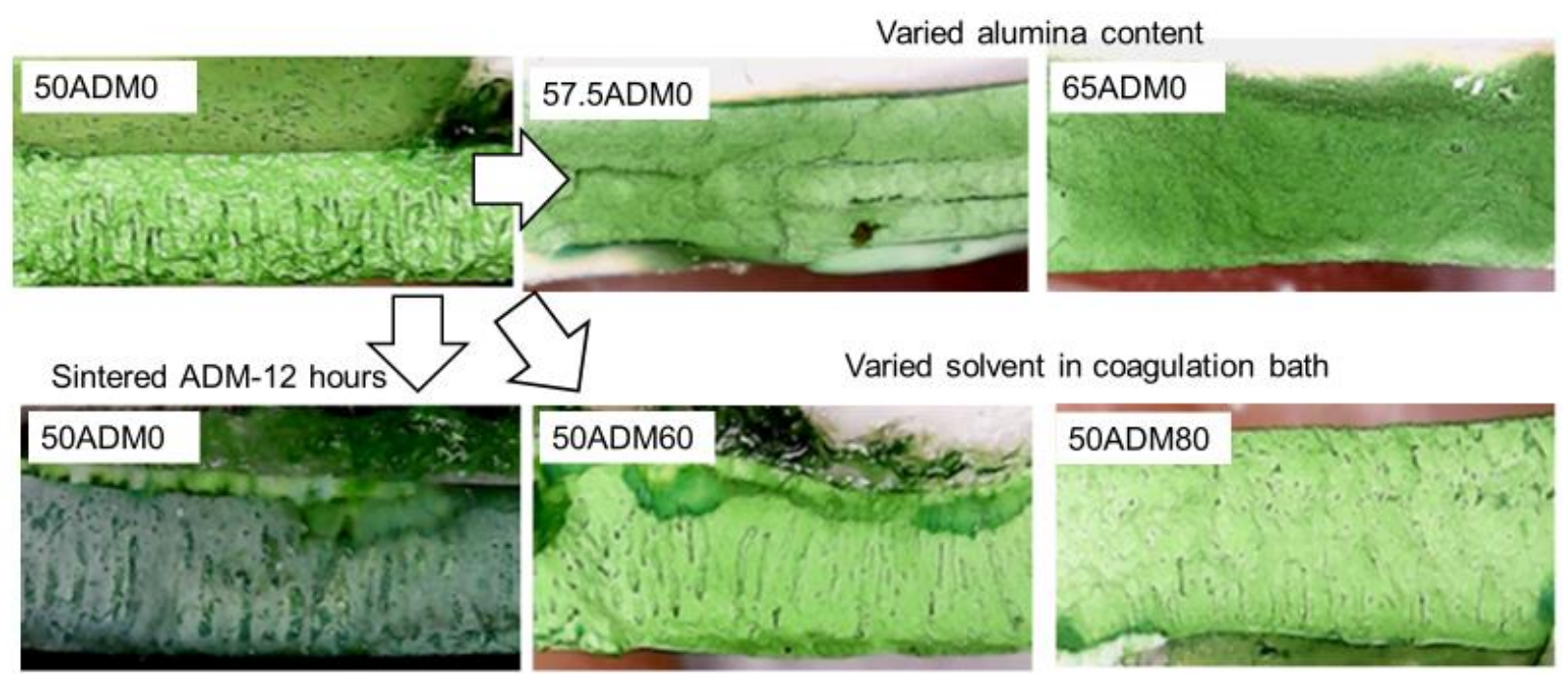

Fig. 1: Digital microscopes images of ADMs prepared in this work by varying alumina loading, DMSO content in coagulation bath and sintering temerature from 8 hours to 12 hours.

Polymer composition in the suspension may also affect the suspension viscosity. Interestingly, the results of this work contradicts with that in a previous study by Abdullah [5] that found that an increase of alumina composition and reduction in polymer content had caused the growth of the finger like formation from $28 \%$ to $56 \%$ [6]. The increase in polymer content is also said to enhance the growth of finger like region due to the sensitivity of polymer binder towards phase inversion process that promotes the finger like structure. Hence, the higher polymer concentration in ceramic suspension the longer length of the finger-like structure. The inconsistent result might be due to the higher amount of alumina in this work, leading to a very viscous suspension that can slow down/inhibit the exchange of solvents as the alumina particles become resistant towards the exchange to occur. The dense structure of 57.5ADM and 65ADM might be beneficial for gas separation as compared to water treatment as the gas separation membranes require a dense structure.

The effects of solvent content in coagulation bath was then investigated. DMSO was added into the coagulation bath to slow down the phase inversion process after the membranes were cast. 50ADM0 was immersed in $0 \%$ non-solvent (pure water) while 50ADM60 and 50ADM80 indicates that the 50ADM membrane was immersed in $60 \%$ and $80 \%$ concentration DMSO, respectively. It can be seen in Figure 1 that as the DMSO content in coagulation bath was increased to $60 \%$, the finger in the membrane became shorter and gradually decreased when the solvent increased up to $80 \%$. The finger-like section did not completely disappeared but the length of the finger gradually decreased and became smaller when higher content of DMSO was added into the bath. The formation of finger occurred at the bottom side of the alumina membrane due to the penetration through the membrane which were open at the bottom. At the outer regions of the membrane, they are observed to consist of a thin sponge like structure.

In this study, the membrane 50ADM0 shows the formation of finger at the bottom part rather than at the top due to the fact that the bottom side is in contact with the water first, earlier than the outer surface and hence the phase inversion as well as the formation of finger initiated from there. This phenomenon is because of the outer surface that is in contact with air resulted in change of the local viscosity due to the solvent that was evaporated and the precipitation of the polymer that was induced by moisture in the air [7]. As the area of viscosity at the outer surface had increased the formation of finger-like structure was inhibited when the casted membranes entered the water bath.

Increasing the solvent concentration to $60 \%$ by adding DMSO (50ADM60) led to the decrease of finger-like macrovoids. The pore size was also decreased and became narrower compared to 50ADM0. This is because of the fact that DMSO was used as both the coagulant and solvent in alumina suspension. During the phase inversion process, the viscosity difference between the suspension and solvent solution decreased as a result of the non-solvent that is not pure water hence the exchange of the solvent and non-solvent did not occur as rapid as when using pure water [8]. Increasing the solvent concentration up to $80 \%$ showed that the formation of finger is less visible and became shorter. Besides that, higher solvent composition in the external coagulant caused longer time for $\mathrm{ADMs}$ to complete its phase inversion process.

The sintering time of 50ADM0 was then increased from 8 hours to 12 hours to observe the effects of sintering time on the membrane morphology. It can be seen that the morphology of ADMs did not show much difference except slightly denser. The densification of sponge like region occurred during calcination resulting in the porosity of membrane to decrease but interestingly the finger macro void was increased. It can be seen that the voids are bigger compared to the membrane sintered at $8 \mathrm{~h}$. This is supported by a study by Abdullah [9], in which they observed longer sintering time caused the polymer binder to be completely removed leaving to formation of large voids.

\subsection{Filtration Performance Study}

Figure 2 shows the filtration performance study for 50ADM0, 57.5ADM0 and 65ADM0. It can be seen that the increase of alumina loading had caused the permeate flux to be decreased. When the alumina loading was increased up to $65 \%$, a reduction of flux up to $60 \%$ was observed. This is consistent with the morphological study conducted earlier in which 57.5ADM0 and 65ADM0 consists of dense structure. This sponge dense structure acts as a flow resistance layer, thus reducing the permeate flux value. As 50ADM0 has finger-like region dominating almost $80 \%$ of the 
total thickness, it is easier for the water to permeate because of lesser resistance. According to Carman-Kozeny model, the increment in water permeation is proportional to the decrease in membrane thickness. In a previous study [8], it was shown that the effective thickness to be applied for this model is by calculating the thickness of the finger-like region only. Hence, it is obvious that 50ADM0 was the only membrane that have effective thickness as it appeared to form a finger like region and thus contributes to higher water permeation flux. Salt rejection performance were also observed to increase up to $40 \%$ when the alumina loading increased. It can be seen from the trend of bar chart that the salt rejection increased when the flux is at its lowest. The trend of the water permeation and salt rejection are inversely proportional in this work.

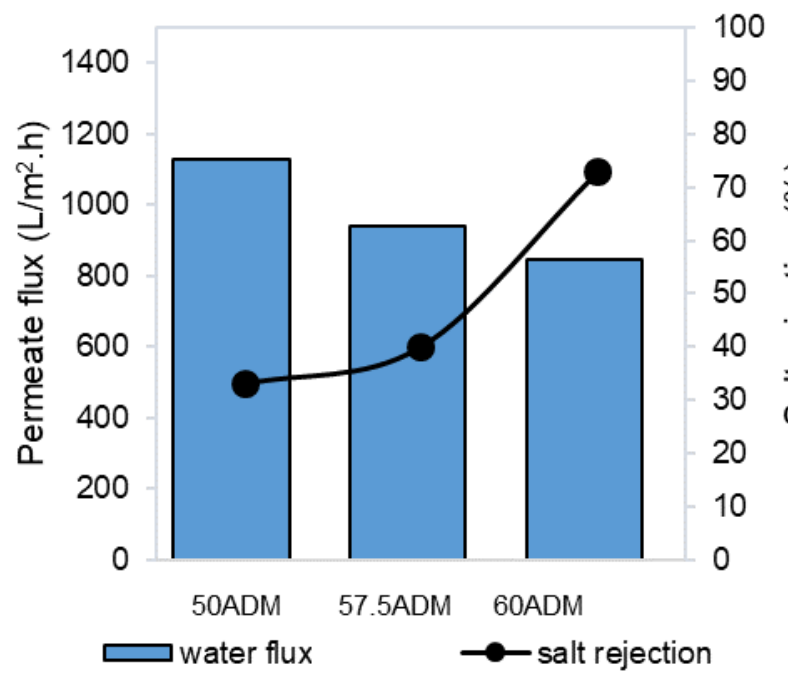

Fig. 2: NaCL rejection study test using different alumina composition 50\%, $57.5 \%$ and $65 \%$

Figure 3 compares the ADMs prepared using various concentration of DMSO in the coagulation bath. It can be seen that the permeate flux was higher when the membrane are immersed at $0 \%$ solvent (pure water) and the lowest flux was observed when $80 \%$ DMSO were added into the coagulation bath. This trend is in agreeing with the morphology of ADMs due to the presence of dense sponge-like region that created a resistance for water to permeate. 50ADM0 that possess finger-like structure and lager voids helped water to permeate easily. Even though, there was a presence of finger-like region on the $60 \%$ and $80 \%$ solvents membrane, they appeared to be shorter and less visible, thus decreasing the permeate flux.

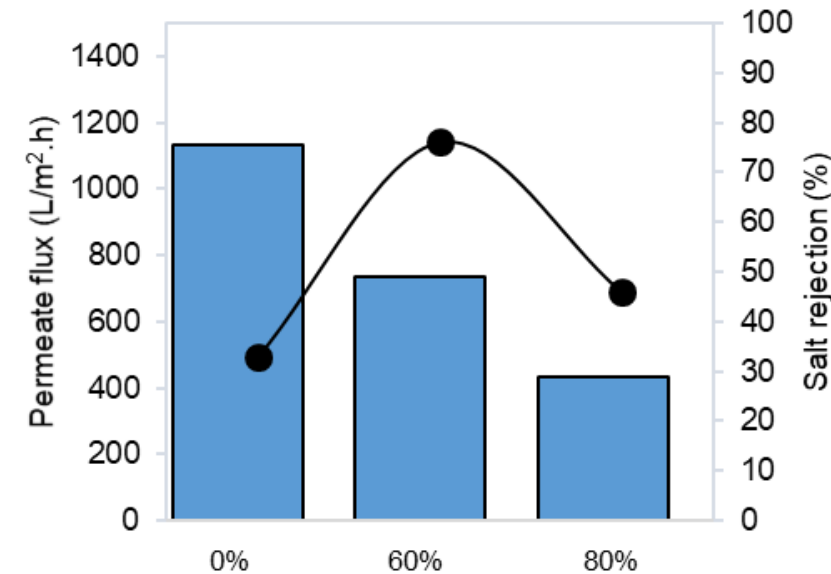

Concentration of solvent in coagulation bath

ऍater flux

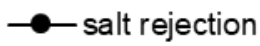

Fig. 3: NaCL rejection study test of membranes prepared in $0 \%, 60 \%$ and $80 \%$ DMSO in coagulation bath

Fig. 4 shows the effect of sintering time towards the $\mathrm{NaCl}$ filtration performance. It can be seen that the longer sintering time can increase slightly the water flux. This might be due to the presence of macrovoids. For salt rejection, there was a significant difference between these two membranes. Longer sintering time led to an increase of salt rejection. Thus, it can be concluded that the longer sintering time is preferred to improve the permeate flux and also salt rejection.

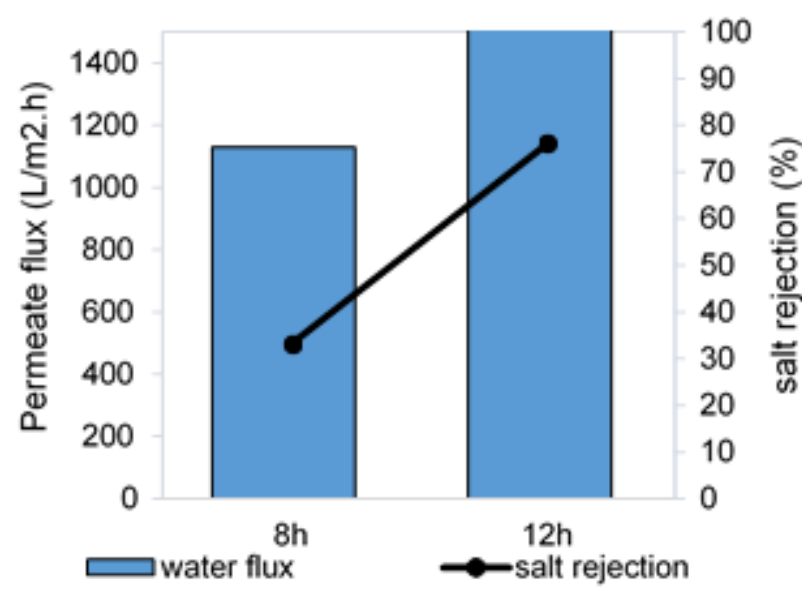

Fig. 4: NaCL rejection study test of 50ADM0 sintered for 8 hours and 12 hours

\section{Conclusion}

In this work, influence of alumina loading, solvent concentration in coagulation bath and sintering time towards the morphology and filtration performance of alumina disc ceramic membranes (ADMs) were investigated. It was observed that the ADM with higher alumina loading tends to form a dense structure. The alumina composition at around 50\% produced a typical asymmetric structure that have better performance in terms of permeance flux and average in salt rejection. The solvent concentration was found to significantly affect the length of the finger-like structure and morphology by reducing the voids which were shortened and caused a decrease in water permeation performance and increased salt rejection. The sintering time had shown to cause the formation of larger void and pores that led to higher water permeate and the effects of rejection on alumina disc with higher sintering time does not show significant effects. In terms of water filtration, ADM that has the highest permeation ability must be chosen for future study 
particularly for desalination purpose. This work has shown that the morphology and filtration performance significantly affected the parameter chosen in the preparation of membrane.

\section{Acknowledgement}

The authors gratefully acknowledge the facilities and support from grant 600-IRMI/DANA/5/3/LESTARI (0029/2016), Universiti Teknologi MARA, Malaysia.

\section{References}

[1] Pichiah. Various fabrication methods of porous ceramic supports for membrane applications. Reviews in Chemical Engineering. 29. (2013). 357-383. 10.1515/revce-2013-0006.

[2] Strathmann, H_, and K. Kock. "The formation mechanism of phase inversion membranes." Desalination 21, no. 3 (1977): 241-255.

[3] B.F.K. Kingsbury, Z. Wu, K. Li, A morphological study of ceramic hollow fibre membranes: a perspective on multifunctional catalytic membrane reactors, Catal. Today 156 (2010) 306-315

[4] Othman, Nur Hidayati, Nur Hashimah Alias, Munawar Zaman Shahruddin, Siti Nurliyana Che Mohamed Hussein, and Aqilah Dollah. "Supported graphene oxide hollow fibre membrane for oily wastewater treatment." In AIP Conference Proceedings, vol. 1901, no. 1, p. 020008. AIP Publishing, 2017.

[5] Abdullah, N. et al., 2016. Preparation and characterization of dual layer thin layer LSCF/Alumina hollow fiber using dip coating and Brush coating technique Ceramics International, 42(10), 1231212322.

[6] Lee, M., Wu, Z., Wang, R., \& Li, K. (2014). Micro-structured alumina hollow fibre membranes - Potential applications in wastewater treatment. Journal of Membrane Science, 461, 39-48.

[7] Lee M., Wang, B., Wu, Z., \& Li, K. (2015). Formation of microchannels in ceramic membranes - Spatial structure, simulation, and potential use in water treatment. Journal of Membrane Science, $483,1-14$

[8] Lee, M., Wang, B., \& Li, K. (2016). New designs of ceramic hollow fibres toward broadened applications. Journal of Membrane Science, 503, 48-58.

[9] Abdullah, N., Rahman, M. A., Othman, M. H. D., Ismail, A. F., Jaafar, J., \& Aziz, A. A. (2016). Preparation and characterization of self-cleaning alumina hollow fiber membrane using the phase inversion and sintering technique. Ceramics International, 42(10), $12312-12322$. 